
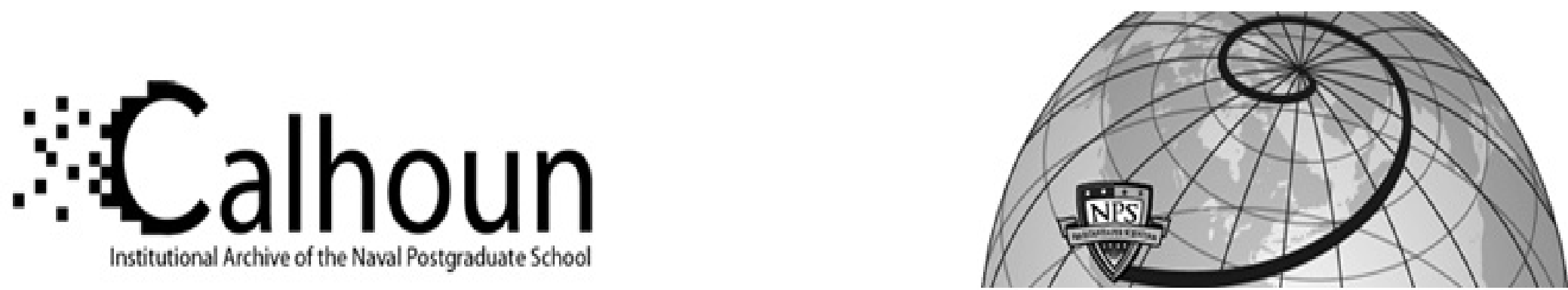

Calhoun: The NPS Institutional Archive DSpace Repository

\title{
On Line Compensation of Heading Sensor Bias for Low Cost AUVs
}

Healey, A. J.; An, E. A.; Marco, D. B.

Healey, A. J., An, E. A., Marco, D. B., " On Line Compensation of Heading Sensor Bias for Low Cost AUVs", Proceedings of the IEEE Workshop on Autonomous Underwater Vehicles, AUV98, IEEE Catalog. Number 98CH36290, ISBN \# 0-7803-5190-8, August 20-21, 1998, Cambridge, Mass. pp 35-42

https://hdl.handle.net/10945/36053

This publication is a work of the U.S. Government as defined in Title 17, United States Code, Section 101. Copyright protection is not available for this work in the United States.

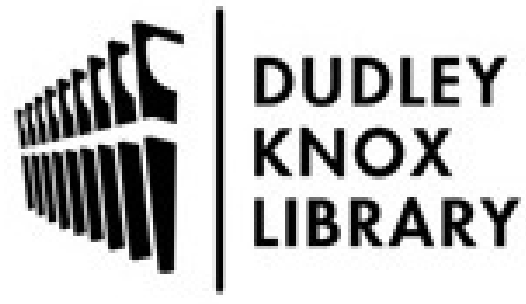

http://www.nps.edu/library
Calhoun is the Naval Postgraduate School's public access digital repository for research materials and institutional publications created by the NPS community. Calhoun is named for Professor of Mathematics Guy K. Calhoun, NPS's first appointed -- and published -- scholarly author.

Dudley Knox Library / Naval Postgraduate School 411 Dyer Road / 1 University Circle Monterey, California USA 93943 


\title{
On Line Compensation of Heading Sensor Bias for Low Cost AUVs
}

\author{
A. J. Healey, ${ }^{+}$E. P. An ${ }^{*}$, D. B. Marco, ${ }^{+}$ \\ *Florida Atlantic University \\ AUV Laboratory \\ +Naval Postgraduate School \\ Center for AUV Research
}

\begin{abstract}
This paper presents a study of the effects of compass bias on navigational accuracy of autonomous underwater vehicles. Low cost vehicle systems utilize a magnetometer, and Doppler sonar for dead reckoning, and a DGPS fix when surfaced. By learning the compass bias from a few DGPS fixes, the navigational errors can be bounded with only a small number of fixes. The study is conducted using the Florida Atlantic University OEX vehicle and a large set of data obtained from a $3.5 \mathrm{Km}$ run including several segments at different headings so that the dependency of compass error on actual heading could be evaluated.
\end{abstract}

\section{Introduction}

Recent progress on autonomous underwater vehicles (AUV) research has provided the ocean community an alternative strategy for undersea exploration. By capitalizing on the existing COTS technology, small AUVs with sufficient onboard intelligent sensing and control can now be manufactured more reliably at lower cost. To push the technological envelope further, multiple small AUVs can be deployed in a co-operative and communicative manner, thereby providing an additional force multiplier for performing cost-effective feedback adaptive search and survey operations. Important AUV applications include autonomous ocean sampling networks, and rapid environment assessment and reconnaissance required for military and humanitarian mine-countermeasures operations.

Among various performance measures for quantifying AUV capabilities, underwater navigation remains an important challenge to AUV researchers $[1,2,3]$. Sampling information is only useful if the AUV's location can be self-registered precisely. While the differential Global Positioning Systems (DGPS) have been widely used in air and land navigation, they are unusable underwater because of the severe signal attenuation and scattering. Without DGPS, long and short baseline sonar, which provide position information via triangulation of beacon signals, and map registration methods can be deployed although it is of interest to see how long underwater ${ }^{1}$

navigation may proceed with dead reckoning alone prior to surfacing for a DGPS fix.

Besides the external position reference signals, inertial navigation system (INS) may independently measure heading and velocity with onboard motion sensors, and in turn therefore estimate the vehicle position by dead reckoning. INS sensors generally have high update rates but drift as a function of time because of sensor scale factor and bias errors. In contrast, position sensors have drift-free error, but the update rates are generally much lower. Error correction in INS systems requires a velocity reference to bound velocity errors, and this is commonly accomplished in small AUVs using a ground locked Doppler Sonar. These sensors generally have different rate throughputs with arbitrary time offsets, and errors arising from these sensors tend to be auto-correlated over different time scales. There are advantages and limitations to these techniques, and it is thus important to devise a scheme to fuse these information sources together in a coherent manner such that the position estimates are accurate and smooth with minimum drift.

One of our ongoing research goals is thus to develop a low-cost, high-precision AUV navigation system by combining COTS components. DGPS fixes are often used to bound error growth, but are unavailable during fully submerged vehicle operations, and the navigation performance in the horizontal plane is predominantly limited by the heading and speed biases. Between the two sources of error, the speed bias -as compared to DGPS has been observed to be substantially the smaller of the two $(0.2 \%)$. In contrast, the heading bias based on the Precision Navigation TCM2 compass often exceeds 5 degrees for some vehicle orientations, and thus it must be minimized through compensation before an acceptable navigation performance can be achieved.

\section{Ocean Explorer (OEX)}

\footnotetext{
${ }^{1}$ Proceedings IEEE AUV98 Workshop on Underwater Navigation , Draper Laboratories, Cambridge Mass. Aug 21-22, 1998
} 


\section{Report Documentation Page}

Form Approved

OMB No. 0704-0188

Public reporting burden for the collection of information is estimated to average 1 hour per response, including the time for reviewing instructions, searching existing data sources, gathering and maintaining the data needed, and completing and reviewing the collection of information. Send comments regarding this burden estimate or any other aspect of this collection of information,

including suggestions for reducing this burden, to Washington Headquarters Services, Directorate for Information Operations and Reports, 1215 Jefferson Davis Highway, Suite 1204, Arlington

VA 22202-4302. Respondents should be aware that notwithstanding any other provision of law, no person shall be subject to a penalty for failing to comply with a collection of information if it

does not display a currently valid OMB control number.

\begin{tabular}{|c|c|c|}
\hline $\begin{array}{l}\text { 1. REPORT DATE } \\
\text { AUG } 1998\end{array}$ & 2. REPORT TYPE & $\begin{array}{l}\text { 3. DATES COVERED } \\
\text { - }\end{array}$ \\
\hline \multirow{3}{*}{\multicolumn{2}{|c|}{ On Line Compensation of Heading Sensor Bias for Low Cost AUVs }} & 5a. CONTRACT NUMBER \\
\hline & & 5b. GRANT NUMBER \\
\hline & & 5c. PROGRAM ELEMENT NUMBER \\
\hline \multirow{3}{*}{\multicolumn{2}{|c|}{ 6. AUTHOR(S) }} & 5d. PROJECT NUMBER \\
\hline & & 5e. TASK NUMBER \\
\hline & & 5f. WORK UNIT NUMBER \\
\hline \multicolumn{2}{|c|}{$\begin{array}{l}\text { 7. PERFORMING ORGANIZATION NAME(S) AND ADDRESS(ES) } \\
\text { Naval Postgraduate School,Center for AUV } \\
\text { Research,Monterey,CA,93943-5000 }\end{array}$} & $\begin{array}{l}\text { 8. PERFORMING ORGANIZATION } \\
\text { REPORT NUMBER }\end{array}$ \\
\hline \multirow{2}{*}{\multicolumn{2}{|c|}{ 9. SPONSORING/MONITORING AGENCY NAME(S) AND ADDRESS(ES) }} & 10. SPONSOR/MONITOR'S ACRONYM(S) \\
\hline & & $\begin{array}{l}\text { 11. SPONSOR/MONITOR'S REPORT } \\
\text { NUMBER(S) }\end{array}$ \\
\hline
\end{tabular}

12. DISTRIBUTION/AVAILABILITY STATEMENT

Approved for public release; distribution unlimited

13. SUPPLEMENTARY NOTES

The original document contains color images.

14. ABSTRACT

This paper presents a study of the effects of compass bias on navigational accuracy of autonomous underwater vehicles. Low cost vehicle systems utilize a magnetometer, and Doppler sonar for dead reckoning, and a DGPS fix when surfaced. By learning the compass bias from a few DGPS fixes, the navigational errors can be bounded with only a small number of fixes. The study is conducted using the Florida Atlantic University OEX vehicle and a large set of data obtained from a $3.5 \mathrm{Km}$ run including several segments at different headings so that the dependency of compass error on actual heading could be evaluated.

15. SUBJECT TERMS

\begin{tabular}{|c|c|c|c|c|}
\hline \multicolumn{2}{|l|}{ 16. SECURITY CLASSIFICATION OF: } & $\begin{array}{c}\text { 17. LIMITATION OF } \\
\text { ABSTRACT }\end{array}$ & $\begin{array}{c}\text { 18. NUMBER } \\
\text { OF PAGES } \\
\text { a. REPORT }\end{array}$ & $\begin{array}{c}\text { 19a. NAME OF } \\
\text { RESPONSIBLE PERSON }\end{array}$ \\
$\begin{array}{c}\text { unclassified } \\
\text { unclassified }\end{array}$ & unclassified & & 7 \\
\end{tabular}


The OEX AUV is a small and untethered autonomous underwater vehicle. Its body length is $7 \mathrm{ft}$ (extendable to $10 \mathrm{ft}$ ) and maximum diameter is $21 "$. The hull is based on a modified Gertler Series 58 Model 4154 with a modular mid-section interface such that multiple payloads can be designed independently. The control surfaces are aft-mounted and of cruciform shape, and are replaceable with larger fins if a $3^{\prime}$ parallel mid-section is inserted. In air, the dry/wet vehicle weighs approximately $700 / 1000 \mathrm{lbs}$, and is designed to be neutrally buoyant. The OEX, which is designed to run from 2 to 5 knots with cruising speed of 3 knots, can execute a preprogrammed mission continuously for 10-12 hours before the onboard $\mathrm{Ni}-\mathrm{Cad}$ batteries need to be recharged. Typical recharge cycle is 12 hours, thereby enabling continuous daily missions with minimal downtime. To increase the operational efficiency, vehicle missions can be programmed using either a wet-matable Ethernet connector in water, or RF ethernet interface in air. In terms of its navigational capability, the OEX houses 1) an acoustic Doppler sonar which measures altitude and vehicle velocity with respect to either water column or ground; 2) a Watson AHRS unit which measures tri-axial attitudes, body rates and acceleration; 3) Precision Nav TCM2 flux-gate compass which also measures the Euler attitudes; 4) a Acupoint/Motorola DGPS/GPS receiver unit; and 5) CTD sensor instrument which measures the conductivity, temperature and depth, and is needed for compensating the sound speed profile.

\section{Compass Calibration - Deviation Table}

This section presents our calibration procedures and results obtained using Ocean Explorer AUV as testbed. The calibration process involves the use of TCM2 compass and KVH single-axis fiber-optic gyro. Selfcalibration of the compass and gyro was first carried out, and then followed by cross-comparative analysis between the heading and gyro measurement.

A program embedded in the TCM2, called mpcal, was used to calibrate the compass. This program takes in 3-axis magnetometer field measurement data collected during which the vehicle is spun slowly about its 3 axes, and then automatically builds a look-up table which is in turn used by the TCM2 to minimize internal hard-iron field effect. The TCM2 also provides feedback in terms of the intensities of the horizontal and vertical field after the calibration process is completed.

It should be noted that the mpcal cannot be used to compensate for any external field anomaly and thus a suitable calibration site must be carefully chosen. Otherwise the deviation table obtained in a noisy environment would then consist of an undesirable offset. The calibration site for the study was chosen to be one of the emptied parking lots located at FAU. Marks were placed on the ground which indicates both the magnetic and true north references. The OEX was transported to the lot using an aluminum cradle with pneumatic wheels.

At the beginning of the procedure, the AUV was oriented toward the magnetic north. Previous calibration was canceled, and then the mpcal program was started. The AUV was first leveled and spun slowly for approximately 2 minutes, at a rate close to $1 \mathrm{~min} /$ cycle; and then tilted about its roll axis to about $12^{\circ}$ and $-12^{\circ}$, while another 2 complete cycles at the same rate were carried out for each of the two tilted positions. The calibration program was then stopped, and the results checked. If the calibration performance was found unsatisfactory, the same procedure would be repeated. The result of the calibration can then be used as a baseline for the second calibration step.

This second step makes use of a single-axis gyroscope as a heading reference with which the TCM2 is compared. This gyro has been repeatedly characterized independently with respect to its static and dynamic performances, and results suggest that, within a given time period of compass calibration, it can provide an accurate reference source with high repeatability of scale factor $(<0.5 \%)$ and thermal bias $(<10 \mathrm{deg} / \mathrm{hr})$ errors. Before the calibration was started, the gyro was first mounted inside the AUV such that its main axis was parallel to the compass axis as much as possible, and the initial AUV heading was then set to magnetic north with minimal pitch and roll. At the beginning of each repeating process, the AUV was first held stationary for 2 minutes such that the static bias of the gyro can be estimated. The vehicle was then manually spun at a rate approximately $5 \mathrm{deg} / \mathrm{s}$ for 2 complete cycles before it was stopped at the magnetic north. It is crucial that the magnetic north location is aligned very accurately and the rotation rate is kept as constant as possible so that the scale factor and static biases can be largely removed through data detrending. This process generally repeats at least twice such that independent data can be used to validate the calibration performance.

\section{Deviation Table}

To obtain a deviation table an error, which is the difference between the TCM2 output and the integrated gyro output, is computed and then de-trended as a function of TCM2 output, to a resolution of a degree. It is worth mentioning that the deviation table is useful for correcting the heading error only when the local magnetic signature does not change with time and external signature derived mostly from the earth's magnetic field. Unfortunately, the local field signature often changes with mission profile, 
and thus with time. Examples include electrical current induced fields and shifting in location of ferrous metal and power cable. A more accurate heading for navigation can thus be obtained by adding the deviation table offset and local magnetic variation to the raw TCM2 output. Despite the compensation process, errors can still exist due to residual errors of compass and gyro, human misalignment error to magnetic north, and possible nearby external field anomaly.

\section{Heading Bias Modeling}

The deviation table exhibits a weighted combination of first and second harmonic component with respect to the compass heading, indicating that a significant level of local interference can exist within the OEX. The error consists of an offset, phase angle and magnitude. Thus, an appropriate heading bias model due to internal fielding interference can be given as

$$
\varepsilon=\mathrm{a} \sin \left(\Psi_{\mathrm{m}}+\mathrm{b}\right)+\mathrm{c} \sin \left(2 \Psi_{\mathrm{m}}+\mathrm{d}\right)+\mathrm{e} \ldots
$$

where a, b, c, d and e are the model parameters to be estimated, and $\Psi_{\mathrm{m}}$ is the measured $[4,5,6]$. It was found out that the two-component model is also accurate for a wide range of internal noise sources that are generated by different payloads. The online identification of residual compass bias is therefore necessary, and has been accomplished using an extended Kalman filter.

\section{Navigation using Dead Reckoning}

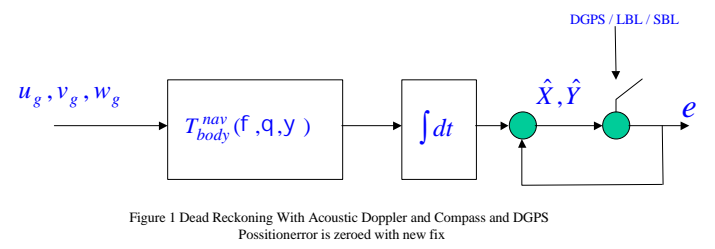

Using dead reckoning as shown in Figure 1, the propagation of a navigation position estimate using the compass angles to rotate the ground referenced velocities as measured by the Acoustic Doppler Sonar is corrected using a reference position measurement from DGPS, LBL, or SBL system. Figure 1 indicates that the estimate is reset to the new position without the ability to adapt to time varying sensor bias.

To learn the compass bias on line and perform suitable balanced corrections on DGPS update, an improved model of the measurements and the vehicle motion model can be used in the setting of extended Kalman filtering. While there are not formal proofs of convergence of the EKF, the EKF will fuse measurements from a vehicle borne Doppler sonar giving vehicle speed over ground, inertial measurements from gyros and a compass -each with biases - and differential GPS when surfaced to estimate 'true' position. LBL or SBL acoustic data, if available, replace the absolute reference role played by DGPS.

One of the problems with fusing data of this type is that inputs are received at non-uniform time intervals and the filter has to deal with local body referenced data as well as globally referenced data. The sensory data do not arrive at the same time, and in particular, DGPS positional data is updated infrequently, while Doppler sonar data arrive at varying times, and sometimes do not give ground locked data at all. Therefore a filtering method is required here to handle asynchronicity in the inputs.

The key to the solution at hand is to define an update rate $(8 \mathrm{~Hz}$.) which is the fastest common nominal rate among the signal channels. Then the filter solution is propagated at that rate where the model based position estimate in the filter is updated with correction gains defined according to whether the data in any channel is new or old. This update 'gain switching', based on currency of data, allows the error co-variances to be corrected by available measurements as they arrive. Error co-variances of those states that are observable only by infrequently updated sensory data, tend to grow almost linearly until the next update. Correction by state observed measurements will always reduce the estimation error co-variances [7]. The method appears to be very satisfactory and the computational burden needs appear to be reasonable on a real time embedded platform.

\section{Continuous Time Model:}

As background, this first section will recap properties of a continuous time filter in which the system model and the output equation is nonlinear

A continuous time model of the vehicle motion appropriate to this problem is taken to be

$$
\begin{aligned}
\dot{x}(t) & =f(x(t))+q(t) \\
y(t) & =h(x(t))+v(t)
\end{aligned}
$$

where $\boldsymbol{x}(\mathrm{t}) \in \mathfrak{R}^{8^{* 1}}$ is the model state. In this system model, $\boldsymbol{f}, \boldsymbol{h}$, are continuous functions, continuously differentiable in $\boldsymbol{x}(t)$, and both $\boldsymbol{q}(t)$ and $\boldsymbol{v}(t)$ are zero mean white noise excitations for the system and measurement models respectively. In this work, the filter states include rate gyro and compass bias, and

$$
\boldsymbol{x}^{\prime}(t)=\left[X, Y, \psi, u_{g}, v_{g}, r, b_{r}, b_{\psi}\right]
$$

In the global reference frame, $\mathrm{X}$ is longitudinal position converted into meters, $\mathrm{Y}$ is latitude position also converted to meters, $\psi$ is the heading angle referenced to 
North, $u_{g}$ and $v_{g}$ are the vehicle speeds over ground in body frame references. $b_{r}$ and $b_{\psi}$ are the biases (assumed constant) associated with both the rate gyro and the compass heading respectively.

The state model is related through the following set of functions representing dynamic relationships between states with assumptions embodying maneuvering models:

$$
\begin{aligned}
& \dot{X}=u_{g} \cos (\psi)-v_{g} \sin (\psi) \\
& \dot{Y}=u_{g} \sin (\psi)+v_{g} \cos (\psi) \\
& \dot{\psi}=r \\
& \dot{u}_{g}=0 \\
& \dot{v}_{g}=0 \\
& \dot{r}=0 \\
& \dot{b}_{r}=0 \\
& \dot{b}_{\psi}=0
\end{aligned}
$$

The model is kinematically based, and, with the last 5 states being integrators, it is possible to obtain unbiased estimations of those states. Measurement bias is handled in the output equation, and high frequency modeling of current states would only be required if significant periodic current components needed tracking.

\section{Output Measurements} through

The output measurements are related to the state

$$
\begin{aligned}
& y_{1}=u_{g} ; \\
& y_{2}=v_{g} ; \\
& y_{3}=\psi+b_{\psi} ; \\
& y_{4}=r+b_{r} ; \\
& y_{5}=X ; \\
& y_{6}=Y ; \\
& \text { or } \\
& \boldsymbol{y}=\boldsymbol{C x}(t)
\end{aligned}
$$

In relation to the above, $y_{1,2}$ are the horizontal velocity components output from the Doppler sonar system which is expressed in vehicle body coordinates. The Doppler measures directly in the body fixed frame. $y_{3}$ is a model of the compass measurement including the compass offset bias. $y_{4}$ is the rate gyro model, while $y_{5,6}$ are the DGPS position data in the global navigation frame, $X, Y$. Bias associated with the Doppler signals is assumed to be negligible.

\section{Implementation}

At some time, $t_{(k+p)}$, in a set of times, $\boldsymbol{T}$, where $k$ is a filter time update, a new measurement data point arrives. The particular sensor, or sensor channel $i$, that provide measurement data at that time are related to the state with the matrix $\boldsymbol{C}$, in a way that is only valid at that particular time. We can express this by

$$
\begin{aligned}
& \boldsymbol{C}\left(t_{i k}\right)=\boldsymbol{C}_{i j}, j=1 \ldots 8, i=i\left(t_{i k}\right) \text { for all } t_{i k} \in \boldsymbol{T} \\
& \boldsymbol{C}\left(t_{i k}\right)=\mathbf{0}, \text { elsewhere }
\end{aligned}
$$

The current state estimate, $\hat{\boldsymbol{x}}\left(\boldsymbol{t}_{\boldsymbol{k}+\boldsymbol{p}}\right)$ is corrected by an amount that depends on the error between predicted and measured value with a gain that provides a set of influence coefficients on each state estimate. The particular sensor, $i$, that provides input to the update is associated with a particular gain set. These gain sets, $\boldsymbol{G}(i, t)$, are computed to provide a least squares error at $t_{k+p}$ based on the particular sensor set being used. Thus it can be said that by considering,

$$
\begin{aligned}
& \boldsymbol{C}\left(t_{i k}\right)=\boldsymbol{C}_{i j}, \mathrm{j}=1 \ldots 8, \text { for all } t_{i k} \\
& \boldsymbol{C}\left(t_{i k}\right)=\boldsymbol{0}, \text { elsewhere }
\end{aligned}
$$

the appropriate gain and covariance corrections are made such that only new information is used in the update. The estimation error co-variances are 'grown' naturally as no new information is available. The usual expressions for the filter state and covariance updates are used.

Additional checking has to be made concerning whether or not new data will be accepted in the filter or whether the filter will propagate the solution without correction. These checks are usually done in relation to the signal to noise ratio of the new data point relative to the current estimated variance of the channel output.

\section{Results}

The data set is a long three loop run where the repeatability of the bias estimate is studied. Here, each loop comprised several headings with each leg run long enough to identify a compass bias. While a correct deviation table for the compass was generated by precalibration, there were residual effects that are noticeable and large enough to cause unwanted positional errors for dead reckoning. Figure 2 shows the path plot. 


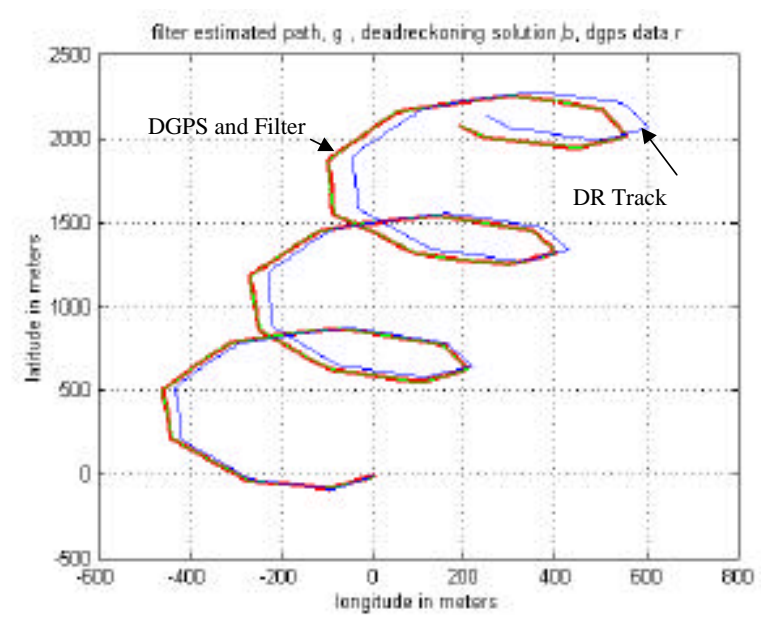

Figure 2 DGPS Path, DR path and Filter Path As Determined by the EKF Using All Available Data .

Compass errors induce a considerable Dead Reckoning Error that is built up during the run. An example of the EKF tracking of the compass heading is given in Figure 3, where it should be noted that errors uring the turn are integrated into positional errors between adjacent segments, and it follows that precise heading measurement round turns is critical.

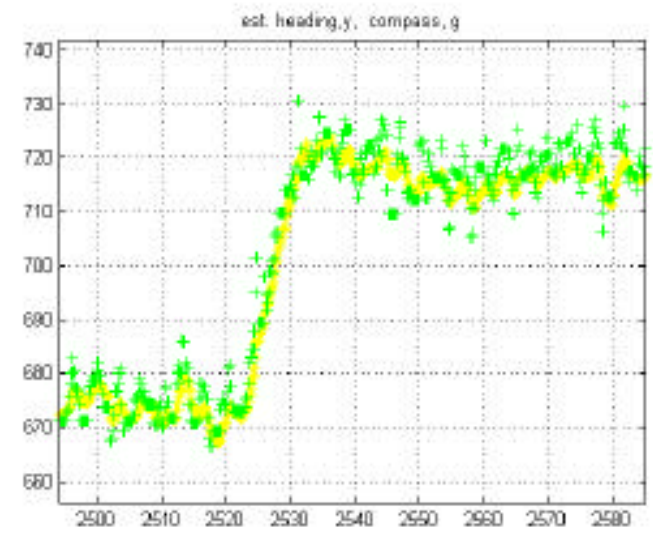

Figure 3 Heading Response During Change. Compass and Filter Heading Estimate.

Compass Errors Round Turns Lead to Unwanted DR Errors in Path

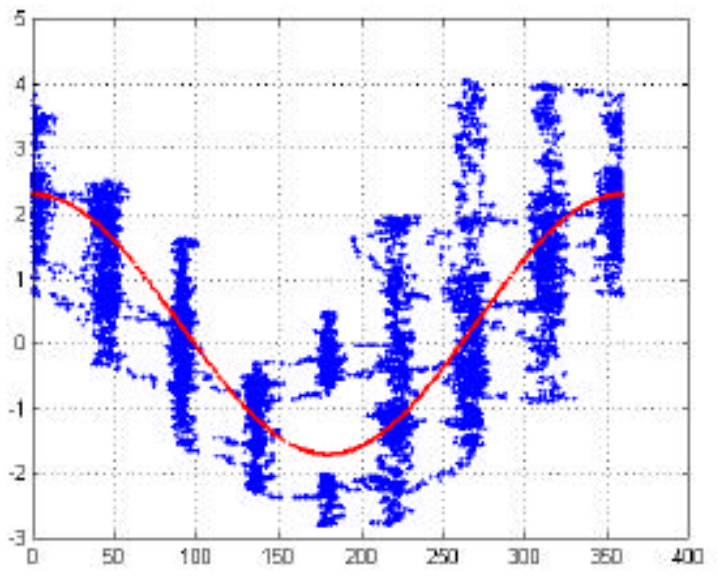

Figure 4. Residual Compass Bias versus Heading. All 3 Loops. Notice that the Bias for the Same Heading does not repeat. The cosine nature is expected from Hard Iron Internal Field Deviations.

\section{Identification of Residual Compass Bias}

The bias identification has several steps. First it is noted that any alignment error between compass and Doppler will produce heading bias. Secondly, compass bias naturally occurs with the present of internal fields in the vehicle. The hard iron errors can be pre-calibrated out of the sensor data through a process of pre-calibration with which the magnetometer coils measure both earth and vehicle fields as the vehicle is rotated. This allows the compass to identify the internal rotating field which it then subtracts from the total field while operating. The residual bias is thus composed of alignment and hard iron error.

Running the filter with DGPS correction, allows the net total bias to be identified. There is a net mean bias that results from alignment. Subtracting alignment errors leaves a zero mean bias where the heading dependent bias has a cosine-like nature. The amplitude is then identified by regression as shown in Figure 4. An amplitude of approximately 2 degrees is still residual and illustrates the importance of performing the deviation table corrections as accurately as possible.

At first sight, it is tempting to formulate the bias as a cosine function with offset and to embed that model into the filter equations. However, this approach leads to a state unobservable filter and was abandoned. Instead, the model above was adopted.

\section{Implementation details}

The 8 state filter has been encoded in ' $\mathrm{C}$ ' and run on a Pentium $100 \mathrm{MHz}$. Processor as well as other 
processors. With careful attention to the computation of the matrix exponential, and the trigonometric functions, we find that computational speeds are in excess of those that would limit control performance in real time [3]

\section{Single Fix Updates}

The filter is also used as a dead reckoning filter without positional corrections. As such, the error covariance of the positional states is used to tell when a new DGPS position is required. This analysis of the data is useful since it allows one to determine the length that dead reckoning solution can proceed prior to the need for a surface command to take a position fix. Adopting this approach, the filter bias estimation is updated from gyro, Doppler, and compass channels until positional error covariances grow to a defined bound. Depending on the bound used, time between fixes can be selected. Figure 5 shows the results of the residual bias estimation response from single fix data, and Figure 6, the resulting bias as a function of heading. Figure 7 shows the convergence properties of the bias estimate. Figures 7 show the response of the bias estimation error covariance which reduces quickly to small values. Figure 8 shows that the position estimation error is reduced each time a fix is made, but which grows between fixes - not always linearly. A bound was set at $400 \mathrm{~m}^{2}$ to need about 7 fixes for the entire run. Figure 9 shows the performance from start for the filter using the first 3 fixes. This illustrates that the bias is quickly learned and the path follows the DGPS data well while the DR track is erroneous.

Figure 10 illustrates that for the experiment conducted and for 20 meters standard deviation error bound, the average time between was 428 seconds. For a 5 meter standard deviation, the average time between fixes was 73 seconds.

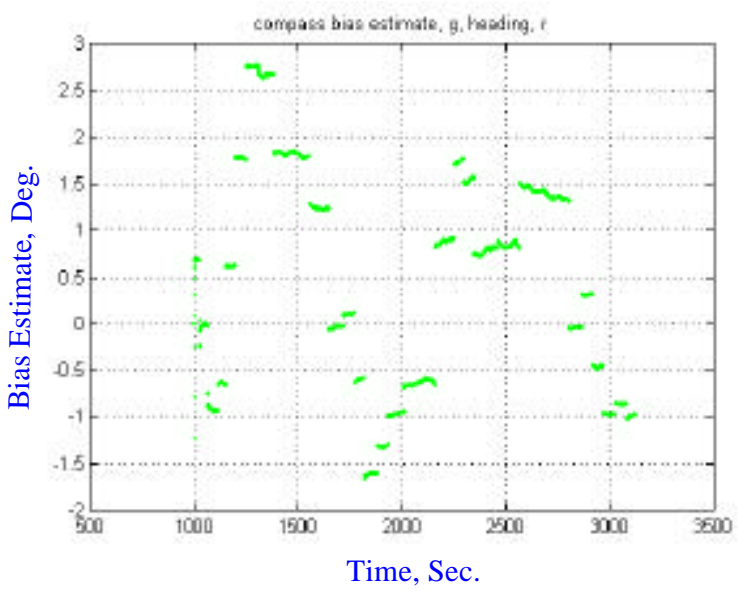

Figure 5 Bias Identification vs Time

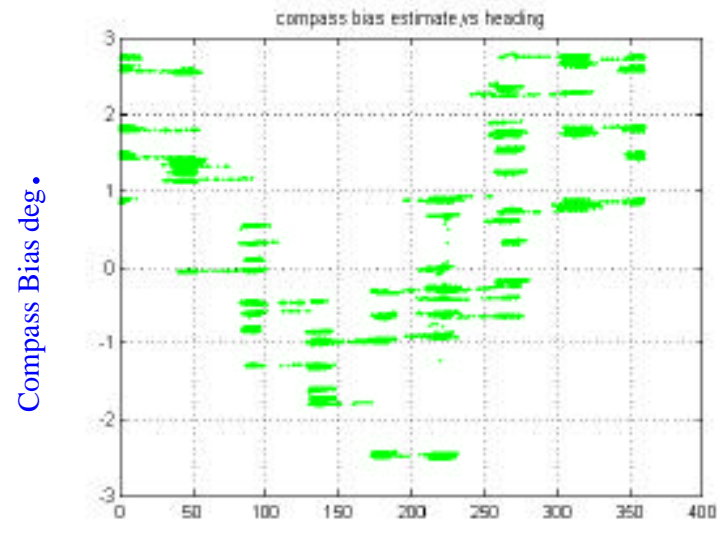

Compass Bias Identified vs. Heading (deg)

Figure 6 Compass Residual Bias vs Heading Single Fixes

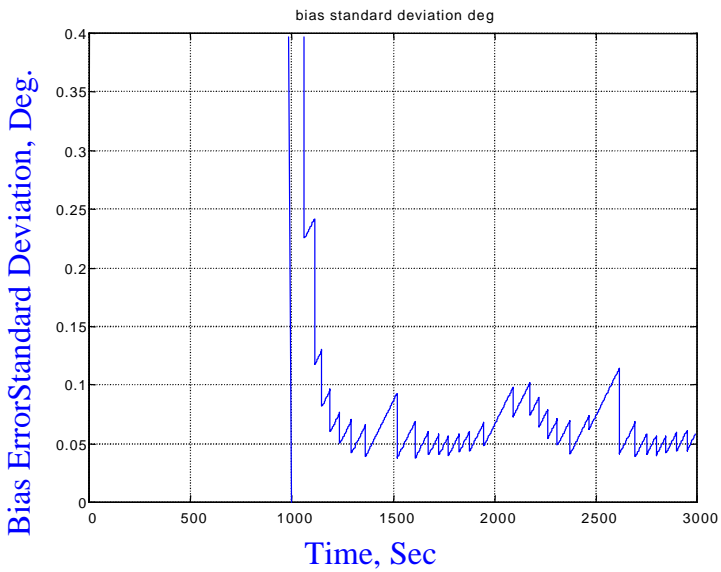

Figure 7 Bias Estimation Error Standard Deviations m.

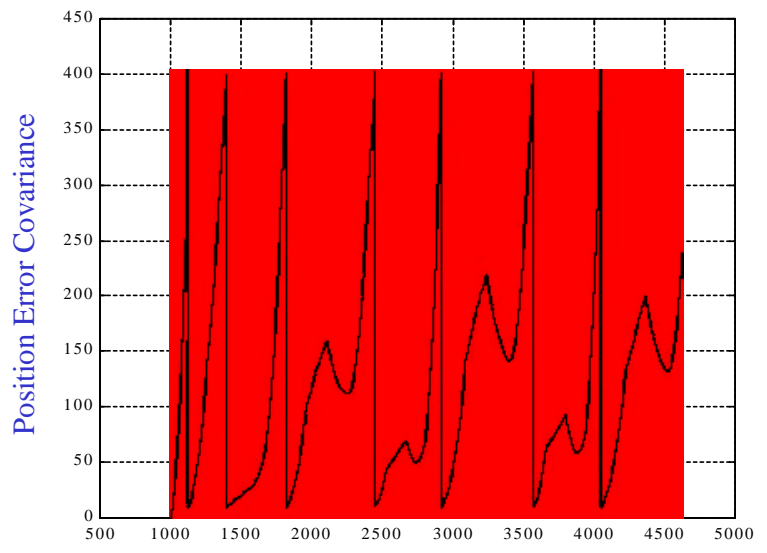

Time in Sec.

Figure 8 Position Error Covariance vs Time With $400 \mathrm{~m}^{2}$ Limit 


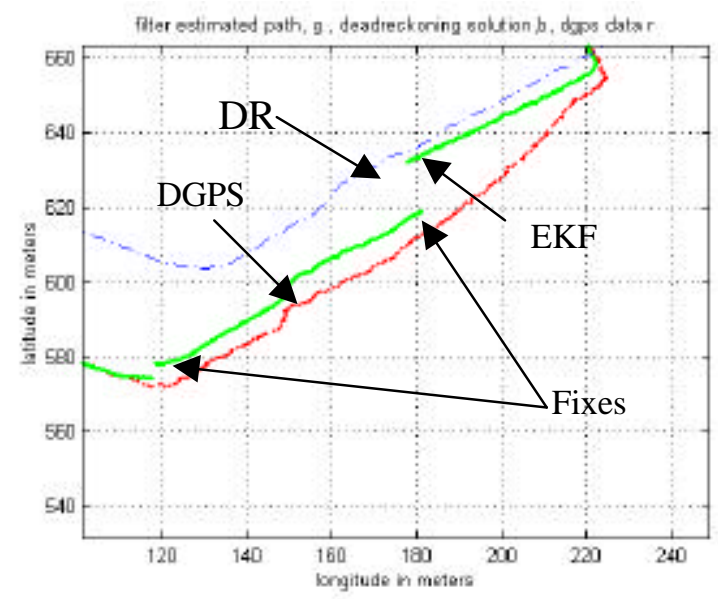

Figure 9 Position Estimation - Dead Reckoning, DGPS data, and EKF Solution

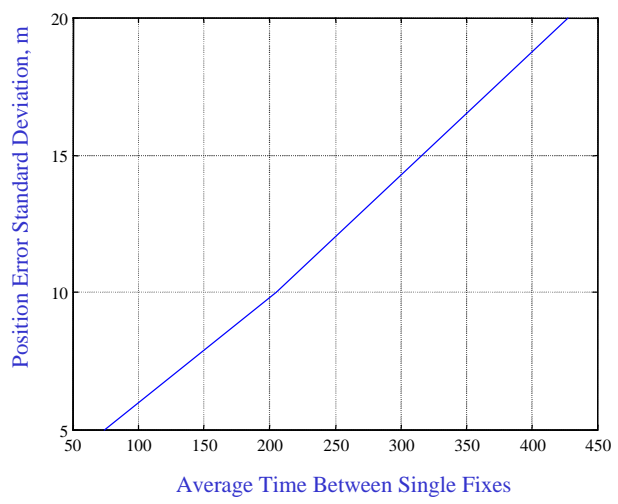

Figure 10 Average Time between Fixes for Different Error Conclusions

Bounds.

As expected, heading bias using a low cost magnetic compass depends on heading itself and can be learned by the EKF using a series of single fixes. DGPS data is therefore necessary to bias learning. The Doppler ground speed data appear to be accurate to within 0.2 percent as compared to DGPS averaged results. Bias model embedding results in unobservable states, and the residual bias must be learned using the simpler formulation proposed here followed by regression analysis on the data.

The magnetometer errors appear to be sensitive to turn rate even though the centrifugal accelerations are low and would not apparently cause biased errors. It is the integrated effects of acceleration induced errors that appear to cause path errors in the dead reckoning solution. For low cost navigation solutions the heading ref is the most critical element in achieving precision.

The 2-D extended filter appears to sufficient and extension to 3-D is not warranted. As the compass bias is not repeatable between loops at the same heading, other fundamental error sources are apparently present.

\section{References}

[1] Session 1, Proceedings of the IEEE AUV 98 Workshop on Underwater Navigation. Draper Laboratories, Aug 20-21, 1998.

[2] An, P. E., Healey, A. J., Park, J., Smith, S. M., " Asynchronous Data Fusion For AUV Navigation Via Heuristic Fuzzy Filtering Techniques ", Proceedings IEEE, Oceans 97, Halifax, Oct. 1997 IEEE CD-ROM 0-78034111-2 http://web.nps.navy.mil/ me/healey/papers/oceans_97.pdf

[3] Smith, S. M., Healey, A. J., McPhail, S., Russel, R., "Lon Talk as a Standard Protocol For Underwater Sensor Platforms", Proceedings IEEE, Oceans 97, Halifax, Oct. 1997 IEEE CD-ROM 0-7803-4111-2 http://web.nps.navy.mil/ me/healey/papers/Lon_Talk.pdf

[4] TCM2 Compass: Compensation of the Errors on the Ocean Explorer AUV, Gabriel Grenon, Edgar An, June, 1998.

[5] TCM2 Compass: Influence of the Variation of the Pitch and Roll Angles on the Heading Angle, Gabriel Grenon, Edgar An, May, 1998.

[6] Gyroscope RD2030: Calibration Experiments and Results, Gabriel Grenon, Edgar An, June, 1998.

[7] A. Gelb "Applied Optimal Estimation", MIT Press, 1986 